\title{
Fluid-Soil-Structure Interaction Phenomena on Vibration Case at Pump Station Building
}

\author{
Hendri Hermawan, Data Iranata, and Djoko Irawan
}

\begin{abstract}
Excitation at low-rise reinforced concrete building had occurred within the first-year post-construction phase. It is found that the structures laying on thick soil layer while performing up to $4 \mathrm{kPa}$ water transport activity. Three approaches have been adopted to investigate the dynamic behavior and the interaction the phenomenon commonly called fluid-soil-structure interaction. Applying the finite element computation to represent the dynamic of the soil-fluid and structure, existing and ideal-fixed base condition are modeled and compared each. It was found that the structure's modes frequencies, much depend on the rigidity of the base and the fluids traffic on the pump station. Time history string of displacements at the arbitrary point shows that the vibration does occurs and it tendentious increase by time.
\end{abstract}

\section{Keywords—Excitation, Coupled, Interaction, Frequency, Finite element, Dynamic.}

\section{INTRODUCTION}

$\mathrm{R}$ apid development of numerical methods in the past decades retrieved new possibilities to conduct sophisticated structural, soil and fluid dynamics simulations. Complex industrial problems will demand simultaneous solver also, such as fluid-structure and soilstructure interaction phenomenon. Principle approaches used to study the phenomenon such Flow Induced Vibration (FIV) for fluid, Finite Element Analysis (FEA) for building/structure, and for the soil model will use $K$ spring approach in each vertical and horizontally direction. The dynamic properties of fluid-soil and how it affects the dynamic behavior of the structure as a whole is the main objectives of this research.

\section{A. Soil Spring}

The concept of spring constant was first introduced by [1]. He modeled flexible foundation, such as raft, to stand on independent discreet spring elements or supports. Proposed a method to estimate the magnitude of the spring constants [2]. His approach, also known as subgrade reaction model, then became popular and commonly used in the design of raft foundation. Looking back into the origin of this concept the modulus or the coefficient of subgrade reaction, $\mathrm{K}_{\mathrm{s}}(\mathrm{x})$, is defined as the foundation pressure, $\mathrm{p}(\mathrm{x})$, divided by the corresponding settlement of the underlying soil, $\mathrm{d}(\mathrm{x})$, i.e.:

$$
K_{s}(x)=\frac{p(x)}{d(x)}
$$

Assuming the soils inside the bulb pressure zone possess are homogeneous, [3] expanded the Wrinkler model into an elastic model and developed the following equation:

$$
K_{S}=\frac{E_{S}}{B \cdot I_{p}\left(1-v_{S}^{2}\right)}
$$

$$
\begin{array}{cl}
\text { Where, } & \\
B & \text { : width of the foundation } \\
E_{s} & : \text { elastic parameters of soils } \\
I_{p} & : \text { the shape factor of the foundation } \\
v_{s} & : \text { poisson ratio }
\end{array}
$$

\footnotetext{
Hendri Hermawan, Data Iranata, and Djoko Irawan are with Department of Civil Engineering, Institut Teknologi Sepuluh Nopember, Surabaya, 60111, Indonesia. E-mail: hendri.hermawan@gmail.com.
}

[4] Suggested providing higher $K_{s}$ at the edges of the raft and smaller $K_{s}$ at the center position. The interpretation of an existing soil layer data is refers to origin data by IPAMKarang Pilang.

\section{B. Structure Dynamic}

Most structures are exposed to dynamic loading during their lifetime. This dynamic load can, for instance, be caused by storms, fluids, earthquakes or rotating types of machinery. The structural response for such a load can be described by the equation of motion called Forced Vibration Damped System [5]. Hence the fundamental equation for forced vibration is:

$$
m\{\ddot{u}\}+c\{\dot{u}\}+k\{u\}=F_{0} \cdot \sin \omega_{n} t
$$

Where,

$\{\ddot{u}\}$ is acceleration vector

$\{\dot{u}\}$ is velocity vector

$\{u\}$ is translation vector

$F_{o}$ is load amplitude

$\omega_{n}$ is load frequency

$t$ is time.

To model the structural parts, an extensive communication had been conducted from formal meeting with IPAM Karang Pilang as well as on a site survey, in order to get the furthest approach to as-built drawings. Consecutive re-drafting the existing building has been done with Solidworks 2017 to get 3D images and to have simulation using solids model. Solidworks is based on finite element method (FEM) and also able to perform dynamic analysis for both linear and non-linear behavior of structures.

The model contains all elements that are considered to affect the structural behavior, reinforced concrete walls, roof, beams and slabs. The non-concrete roof structure is considered to have no contribution to the overall stiffness of the building and is therefore omitted, except as mass. In the modelling process, boundary conditions at the building supports use elastic support which allows rotation and translation in certain value

\section{Flow Induced Vibration}

The initial study of the experiment of vibrations by giving a certain force (in this case fluid) starting from [6] 


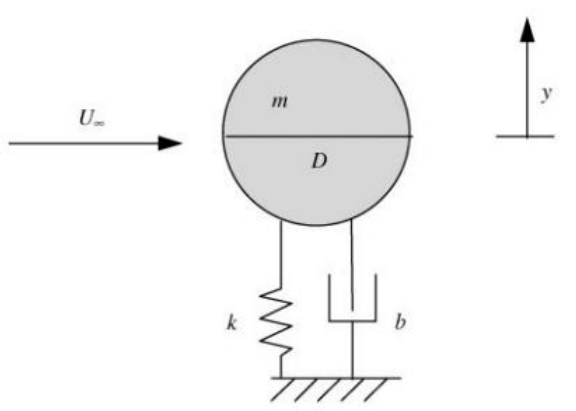

Figure 1. Idealized Flow-Induced Vibration.
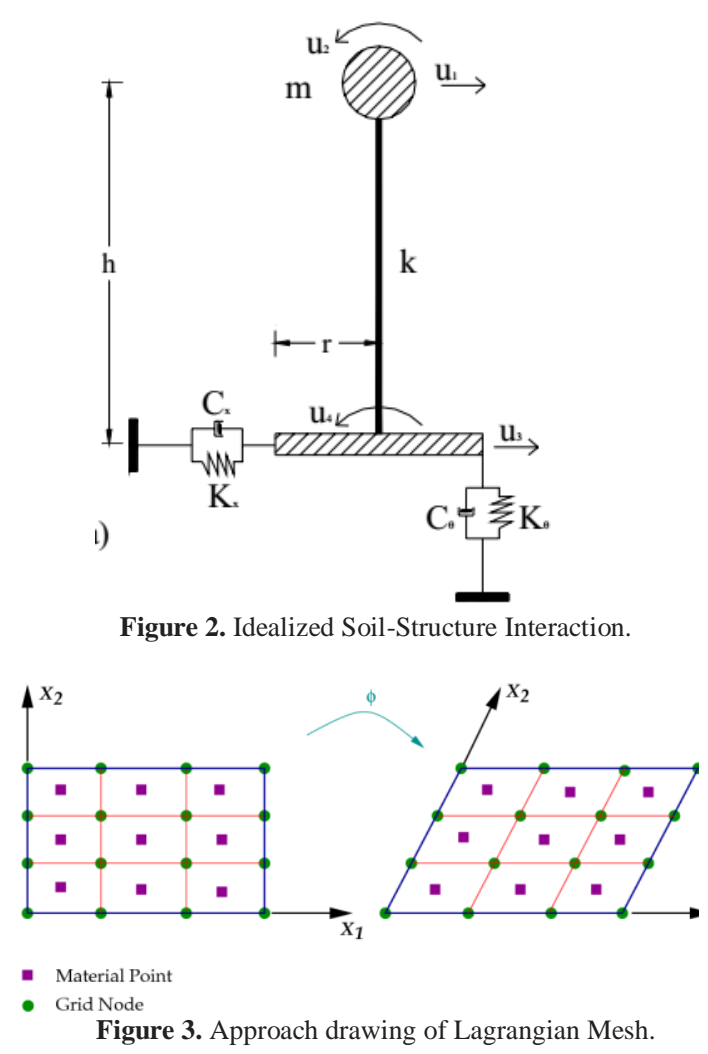

and experiments on free vibrations from [7] determined the viewpoint that the cylindrical shape experienced significant vibrations only when frequency characteristic shedding resonated with frequency natural structural, hereinafter referred to as "lock-in". The experimental results from [8] presented significant examples of static flow-induced vibration without "lock-in". These results indicate that the "lock-in" framework does not accurately describe the overall dynamic behavior, especially when the ratio between cylinders vs fluid masses becomes relatively small.

\section{Coupled-Field Analysis}

A coupled-field analysis is an analysis that takes into account the interaction (coupling) between two or more disciplines (fields) of engineering. The procedure for a coupled-field analysis may divided into two distinct methods sequential and direct, this paper closely using sequential method. Which described as two or more sequential analyses to a different engineering field.

In the simplest understanding is to apply results from the first analysis as loads for the second analysis. However, for coupling situations which do not exhibit a high degree of nonlinear interaction, the sequential method is more

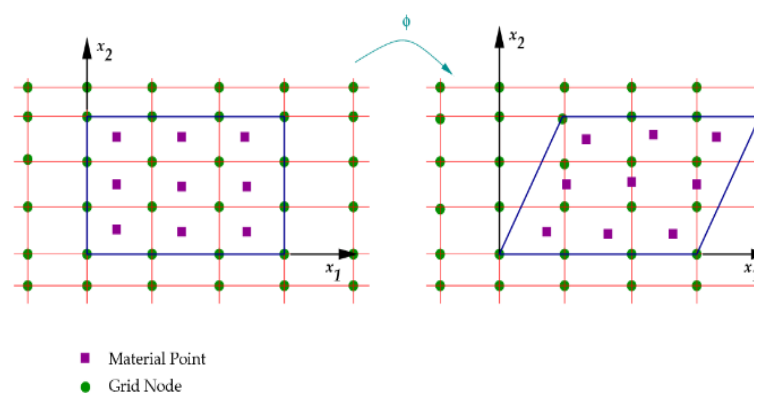

Figure 4. Approach drawing of Eulerian Mesh.

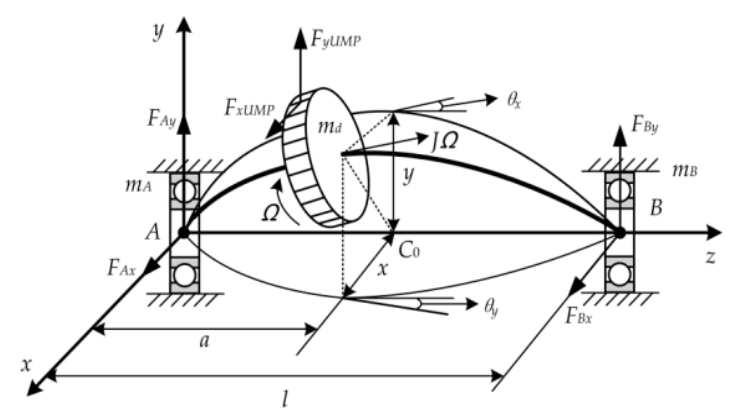

Figure 5. Vibration model of the shaft element [15].

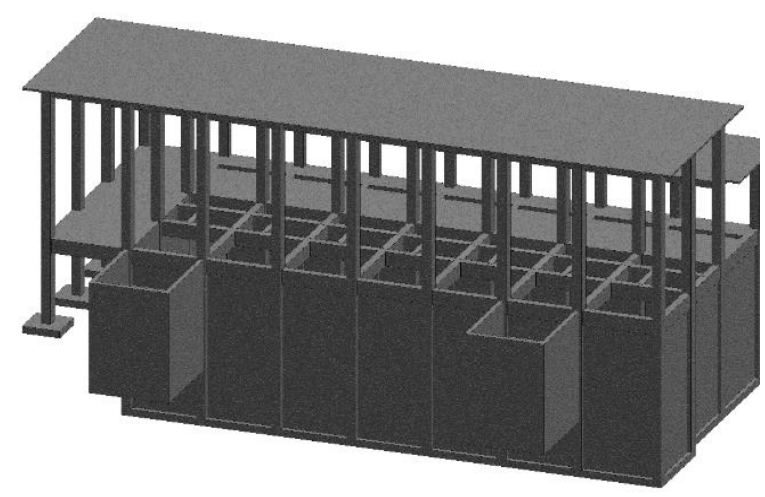

Figure 6. 3D isometric view of pump station.

efficient and flexible. It is known that performing the two analyses independently of each other is reliable also [9].

\section{E. Coupled Soil-Structure}

The classical analysis of soil-structure interaction aims at replacing the actual structure by an equivalent simple oscillator supported on a set of frequency-dependent springs and dashpots accounting for the stiffness and damping of the soil [10].

The system studied is shown in Figure 2 it involves a simple oscillator on a flexible base representing a single story structure, or a multi-story structure after a pertinent reduction of its degrees-of-freedom. A number of different approaches are available to formulate and solve the equations of motion for a soil-structure interacting system. The improvement by [11] is described as the basic equations in solving the horizontal rocking vibration problem of a building-foundation system.

\section{F. Coupled Fluid-Structure}

The distress of a concrete building is affected by several parameters, such as the compressibility, water hammer of the water and lead to various rotor yet fluid dynamic interactions which can be incorporated in the general term 
TABLE 1.

INITIAL PROPERTIES OF THE CONCRETE ELEMENTS

\begin{tabular}{|c|c|}
\hline Mass Density & $2400 \mathrm{~kg} / \mathrm{m} 3$ \\
\hline Poisson's Ratio & 0.205 \\
\hline Concrete Strength & $40 \mathrm{Mpa}$. \\
\hline Elastic Modulus & $2.3 \mathrm{e} 10 \mathrm{Mpa}$ \\
\hline
\end{tabular}

TABLE 2.

INITIAL PROPERTIES OF THE SOIL FOR MODELING.

\begin{tabular}{ccccr}
\hline \hline Depth $(\mathbf{m})$ & $\mathbf{N}$ & Soil Type & $\mathbf{k}_{\mathbf{V}}(\mathbf{k N} / \mathbf{m})$ & $\mathbf{k}_{\mathbf{H}}(\mathbf{k N} / \mathbf{m})$ \\
\hline 0 & 0 & silty & 0.0 & 0.0 \\
-2 & 4 & silty & 20734.5 & 13200.0 \\
-4 & 4 & silty & 20734.5 & 14520.0 \\
-6 & 7 & silty & 26954.9 & 17160.0 \\
-8 & 8 & silty & 29028.3 & 19800.0 \\
-10 & 12 & silty & 37322.1 & 17820.0 \\
\hline \hline
\end{tabular}

TABLE 3.

PARAMETERS FOR FLUID AT $300^{\circ} \mathrm{C}$

\begin{tabular}{lr}
\hline \hline Density & $\mathbf{9 9 5 , 7 7 ~ k g / m 3}$ \\
\hline Visc. Dynamic & $7,91 \times 10-4 \mathrm{~kg} / \mathrm{sec}-\mathrm{m}$ \\
Bulk Modulus & 215013,5 \\
\hline \hline
\end{tabular}

TABLE 4.

NLTH ANALYSIS SETUP

\begin{tabular}{ll}
\hline \hline Analysis type & Non-Linear TH \\
\hline Integration & Direct - Hilber Hughes \\
Initial Cond. & Cont. from NL static \\
TH Motion type & Transient \\
Damping Prop. & Proportional \\
Time Step & $4 s$ \\
Fixture & Fixed and Spring \\
Rotation Freq & $19,17,16,14 \mathrm{~Hz}$ \\
\hline \hline
\end{tabular}

TABLE 5.

NODAL ROTATION (RAD) BETWEEN BEAM AND FLOOR.

\begin{tabular}{ccl}
\hline \hline Node & Rotation & \\
\hline 1 & $2.923 E-06$ & Rad \\
2 & $1.236 E-05$ & Rad \\
3 & $7.695 E-06$ & Rad \\
4 & $7.314 E-06$ & Rad \\
5 & $8.386 E-06$ & Rad \\
6 & $1.134 E-05$ & Rad \\
7 & $1.18 E-05$ & Rad \\
8 & $5.688 E-06$ & Rad \\
\hline \hline
\end{tabular}

"fluid-structure interaction", the possible existence of effect of surface (sloshing) waves, and the selection of an appropriate upstream boundary condition to represent the infinite extent of the reservoir in the upstream direction. The impact of these factors has been investigated in the past [12] analytically, numerically, or even experimentally as it will be briefly discussed in the sequence.

In order to cope with fluid-structure interaction problems, two approaches have been developed; the Eulerian approach and the Lagrangian approach. The Eulerian element formulation allows analysis of a medium which undergoes major deformation by mesh distortion techniques. The Eulerian-Lagrangian interaction formulation allows the eulerian material model to be analyzed through a Lagrangian, such as capturing the phenomenon of extreme deformation of fluid flow. As is seen on Figure 3, the short brief of Lagrangian Mesh are brought below:

a. Nodal coordinates of the Lagrangian move according to the material.

b. The material coordinates of the material point are not changing with time.

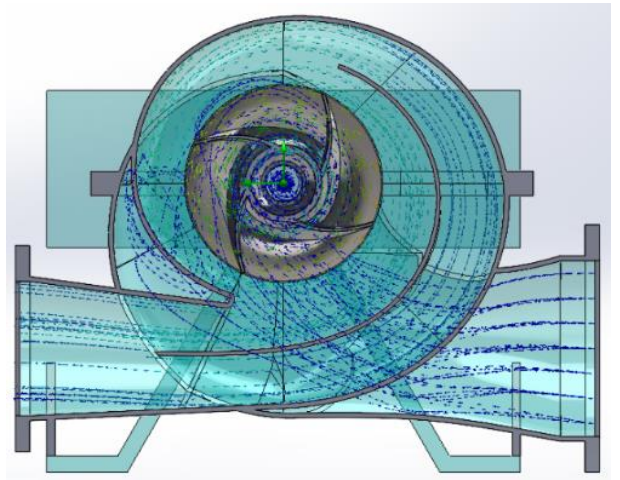

Figure 7. CFD analysis on centrifugal pump.
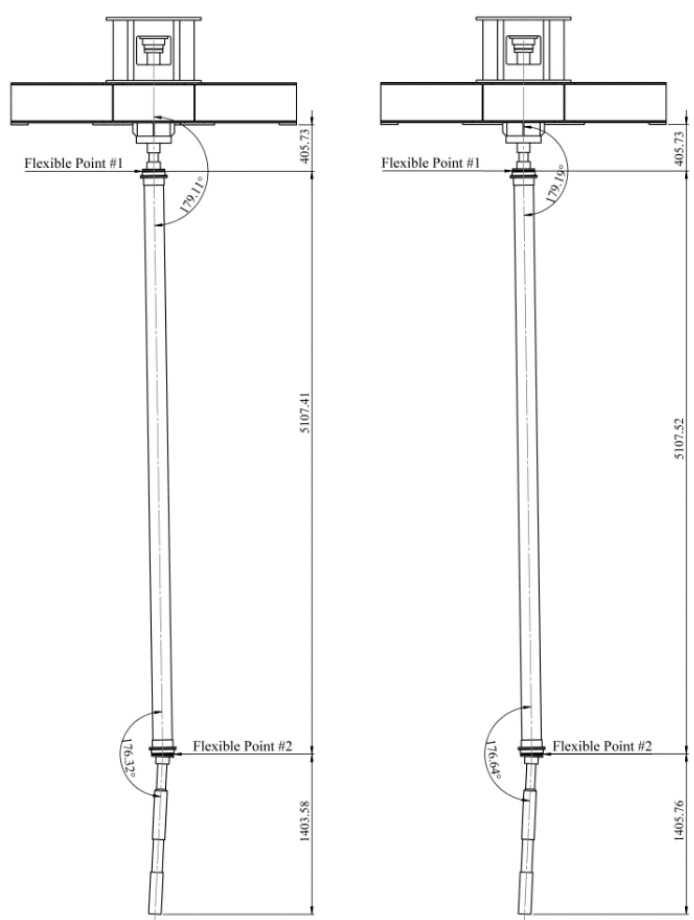

Figure 8. Deflected shape of vertical pump.

c. There is no material that moves through/between elements.

d. 4 quadratic points of the element remain to coincide with the points of the material.

e. The capital limit remains on the boundary when initial defining. Therefore, boundary conditions and interface conditions are easy to apply.

f. Extreme mesh distortion can occur because the mesh deforms together with the material

As is seen on figure 2.7, the short brief of Eulerian Mesh are brought below:

a. Nodal coordinates of Eulerian fix / fixed and coincide with spatial points. The spatial coordinates of material points vary over time.

b. Material flows through/between mesh.

c. The point of the material at the 4 quadratic points of the element changes over time. This makes it more difficult because of dealing with material that depends on the order of time.

d. The material limits and limits allow it not to coincide. Therefore, boundary conditions and interface conditions are difficult to implement.

e. There is no mesh distortion because the mesh remains in space. However, the domain that needs to be modeled is bigger because we don't want the mesh body to leave the domain. 


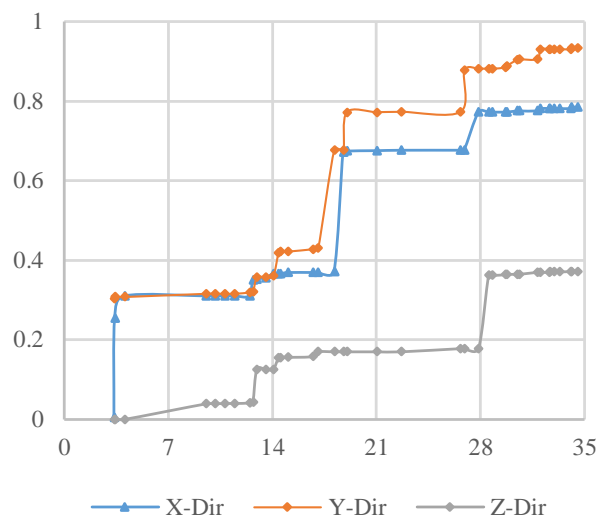

Figure 9. Frequency (Hz) vs Mass Participation (\%) of Fully Fixed base building.

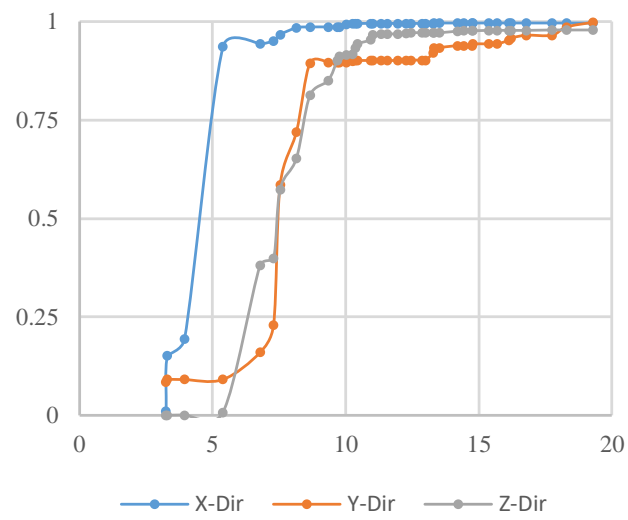

Figure 10. Frequency (Hz) vs Mass Participation (\%) of spring base building.

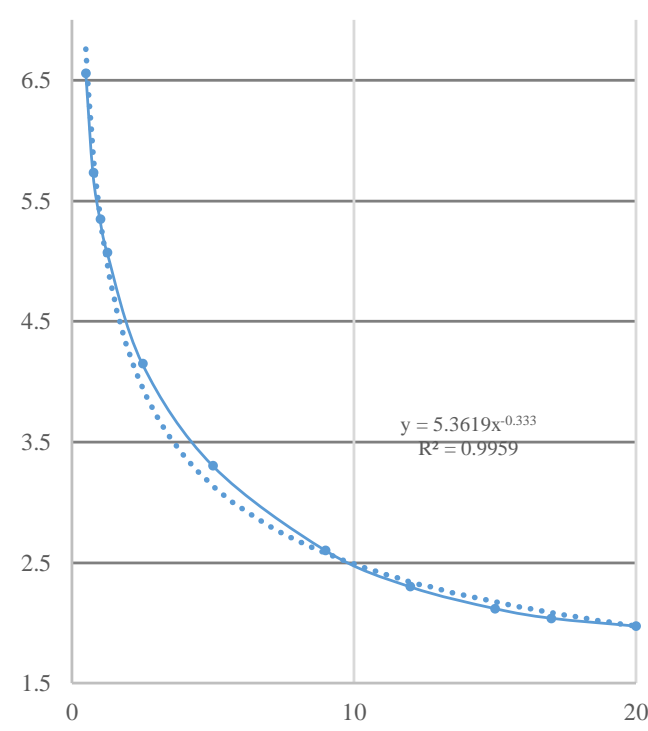

Figure 11. Magnitude of $\mathrm{K}_{\text {spring }}$ vs Rotational magnitude of the shaft.

\section{G. Rotor Dynamics}

A very small amount of unbalance may cause severe problem in high speed rotating machines. The vibration signature of the overhung rotor (as discussed in this paper) is totally different from the center hung rotors. Rotor unbalance is the mostly common reason in machine vibrations [13], the vibration caused by unbalance actually destroy critical parts of the machine, such as bearings, seals, gears and couplings.

Rotor unbalance is a condition in which the center of
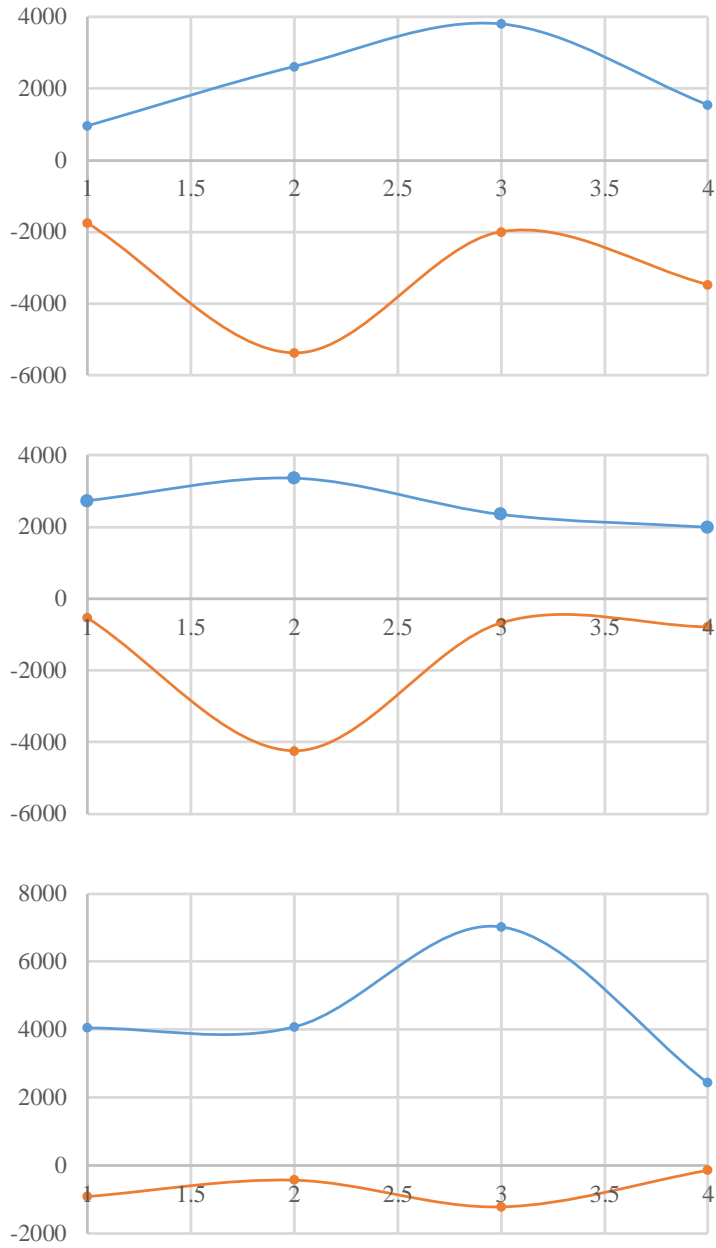

$\longrightarrow$ Max Force Z-Dir $\longrightarrow$ Min Force Z-Dir

Figure 12. Fluid Pressure (Bar) vs Force (N) on Centrifugal Pump.

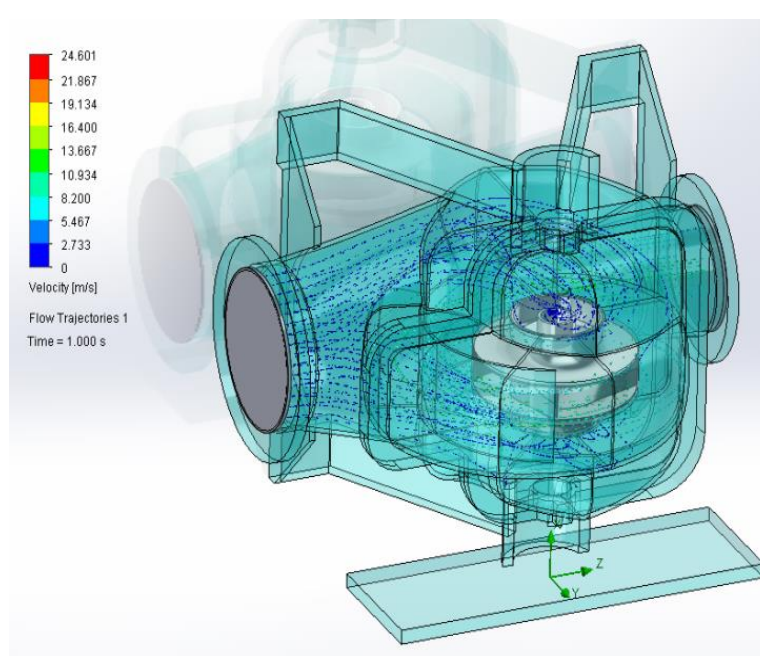

Figure 13. Fluid flow visualization from CFD analysis.

mass of a rotating assembly, typically the shaft and its fixed components like disks and blades etc. is not coincident with the center of rotation. In practice, rotors can never be perfectly balanced because of manufacturing errors such as porosity in casting, non-uniform density of material, manufacturing tolerances and gain or loss of material during operation. As a result of mass unbalance, a centrifugal force is generated and must be reacted against by bearing and support structures [14].

Based on Newton's second law, the equations of 


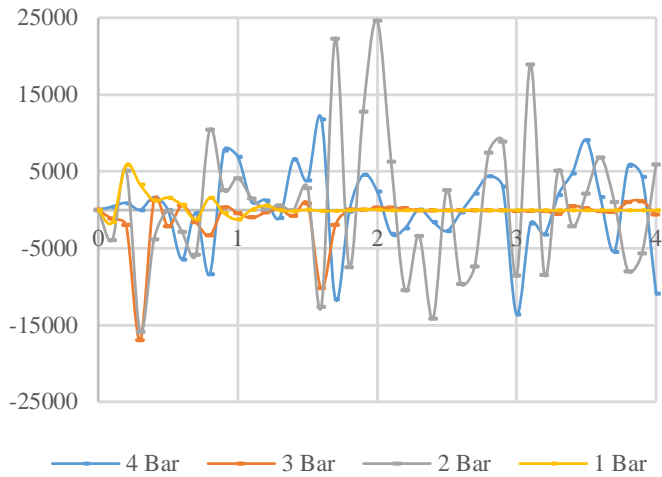

Figure 14. Time (s) vs Force Resultant (N) affect by the Unbalance rotation.
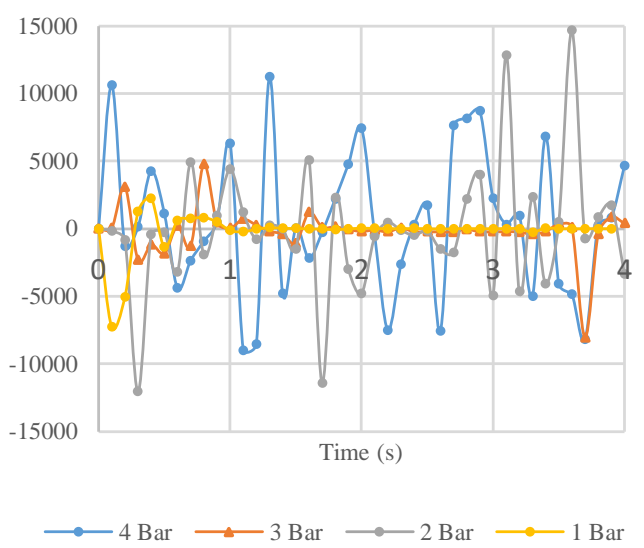

Figure 15. Time (s) vs Force Resultant (N) affect by the Unbalance rotation.

motion (3) can be derived into rotor bearing system as below [15]:

$$
M \ddot{u}^{T}+(\Omega J+C) \dot{u}^{T}+K u^{T}=F
$$

Where $M$ is the mass matrix, $J$ is the gyroscopic matrix, $K$ is the stiffness matrix, and $C$ is the damping matrix. Finally proceed to defining $F$ is the sum of the unbalancemass $F_{1}$ and ball-bearing force $F_{2}$.

$$
F_{1}=\left[\begin{array}{c}
m_{d} e_{0} \Omega^{2} \cos \Omega t \\
0 \\
0 \\
0 \\
m_{d} e_{0} \Omega^{2} \sin \Omega t \\
0 \\
0 \\
0
\end{array}\right] \quad F_{2}=\left[\begin{array}{c}
0 \\
0 \\
F_{A X} \\
F_{B X} \\
0 \\
0 \\
F_{A Y} \\
F_{B Y}
\end{array}\right]
$$

Here some brief explanation of the Figure 5 [15] and equation (4):

$m_{A}$ and $m_{B}$ : mass of the bearings

$m_{d}$ is the mass of the rotor

$a$ : distance between the rotor and the left bearing.

$l$ for length of the shaft

$x, y$ is the position of rotor in coordinate system

$\theta x, \theta y$ is the rotational angles of rotor

And then $e_{0}$ is the mass eccentricity of rotor

$\Omega$ is the rotating speed of the rotor

$\mathrm{J}_{\mathrm{d}}$ is the moment of inertia of rotor

$\mathrm{J}_{\mathrm{p}}$ polar moment of inertia of rotor

$E$ is the Young's modulus of the shaft

$I$ is the moment of inertia of shaft

$c$ is the damping of rotor

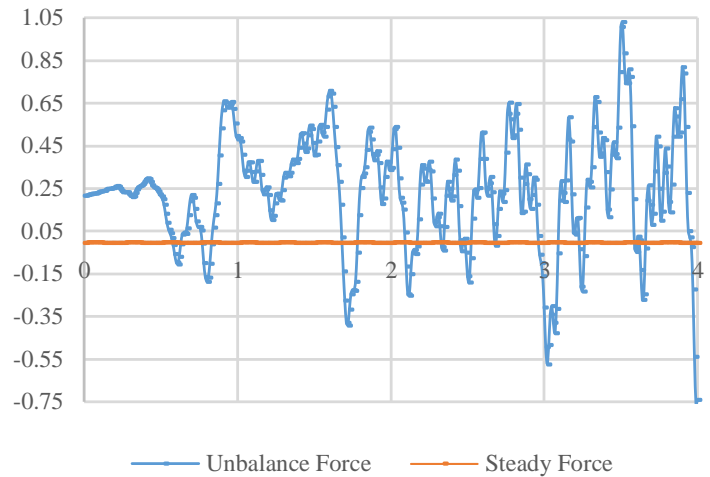

Figure 16. Time (s) vs Beam X-deflection ( $\mathrm{mm}$ ) affect by the Unbalance rotation.

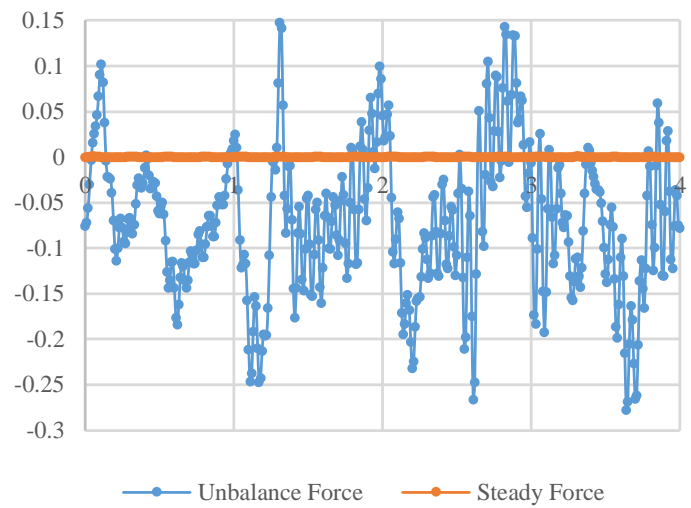

Figure 17. Time (s) vs Beam Y-deflection (mm) affect by the Unbalance rotation.

$c_{b}$ is the damping of bearings

\section{METHOD}

This vibration phenomena retrieve an engineering intuitive prediction for the root-cause of it, and it is widely known that every rotating equipment ideally creates sinusoidal wave. And particularly these rotating equipment are transferring massive fluids with certain range of pressure value. For the soil-structure interaction, it is concluded (and refer to existing drawing) that the building sub-structure system does not use piling material. Hence it is permissible to assume that the structure is stand on foundation with non-linear rigidity aspect.

Here with below are quick recap of how the methodology will flow:

1. Static and Modal analysis: using specimens of 1 pcs fixed-base building and 7 pcs spring-base buildings.

2. Computational Fluid Dynamics analysis: using 4 specimens of different pressure bar, 1 to $4 \mathrm{kpa}$

3. Operational Deflected Shape analysis: using 8 specimens of deflected shape (from step 1) and each will rotate within different frequencies (from step 2)

4. Non-Linear Time History analysis: using 1 specimen of 3D concrete building model which will be loaded with un-balanced force from step 3 .

The representation of the building can be seen at Figure 4 , it is the $3 \mathrm{D}$ isometric-view for pre-analysis phase.

\section{A. Static and Modal Analysis}

This analysis holds basic purpose to determine the alternating of the geometry of the building. Static analysis 


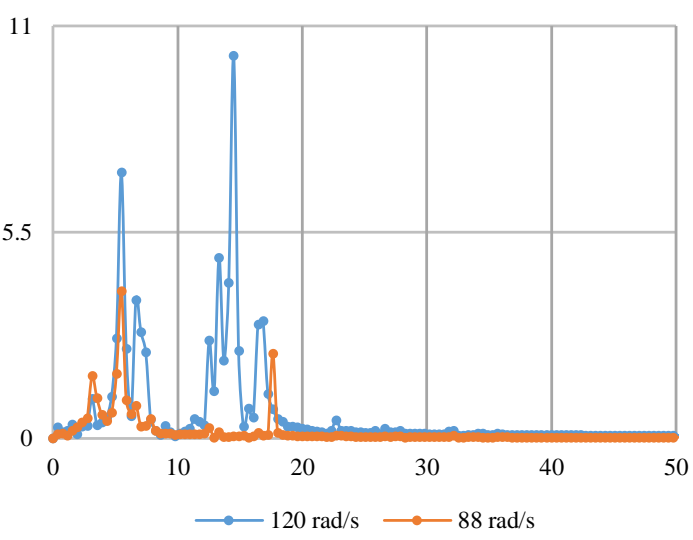

Figure 18. Frequency (Hz) vs Beam deflection (mm) analyzed using Fourier analysis.

(calculated in different spring condition) will producing a graph relationship between soil spring values with the rotation of the vertical pump's geometry. Table 1 show the definition of concrete modeling value, while table 2 describe hand-calculation of $K_{\text {spring }}$ value at depth of $10 \mathrm{~m}$ according existing condition.

\section{B. Computational Fluid Dynamics (CFD) Analysis}

CFD analysis is a branch of fluid mechanics that uses numerical analysis and data structures to solve and analyze problems that involve fluid flows are used to perform the calculations required to simulate the interaction of liquids and gases with surfaces defined by boundary conditions. Figure 7 viewing the boundary environment used in this paper.

\section{Operational Deflected Shape (ODS) Analysis}

This ODS analysis is the continuity from the output shape of static analysis. Every geometric rotational value results from the static analysis shall be correspondent to this analysis, applied as the vertical pump's misalignment. The output of this analysis will be considered as the vibrational force wave-like. Which will be the main external force for next harmonic loading at dynamic analysis. Figure 8 revealed how the parts of vertical pump deflected each to one another, and this shape will be the initial setup for the motion analysis in Solidworks 2017.

\section{Non-Linear Time History (NLTH) Analysis}

This NLTH analysis will take the ODS analysis output as string data force within specific windowing time. This analysis will conducted using FEM software SAP2000 (compacted in Table 4), particularly focused on the performance of NLTH Analysis at the pump station building. The desired outcome from ODS analysis will be take on both conditions fixed and spring base, each conditions shall be represented by force-time graph relations

\section{RESULTS AND DISCUSSION}

The result in Figure 9 describes how the building response within fixed base, it is shown that mass cumulative participation is considered low or far below $80 \%$. It means that the natural frequencies of the building unlikely, not excited by any forced wave frequencies.

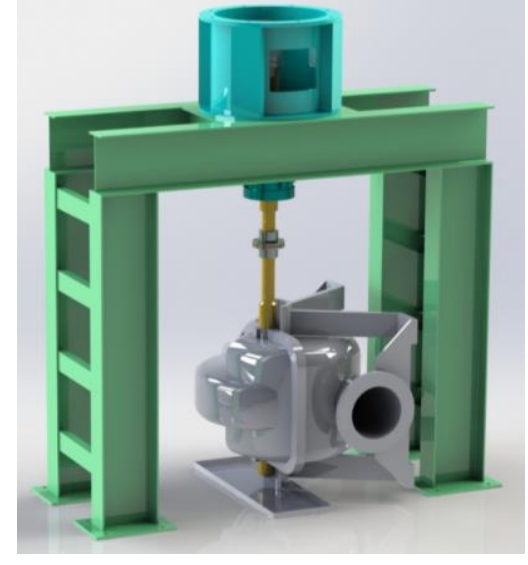

Figure 19. Recommendation to modify the configuration of vertical pump.

However the appearance of graph from Figure 10, can be seen that the mass cumulative participation reach above $80 \%$ in all direction with different frequency value.

Next result is the static analysis, which intended to find how the building response in ideal condition, and comparing the result of it into another elastic base conditions. This means to produce non-dimensional value relationship with of other modal analysis results. Table 5 shows the vertical pump center line's rotation at various vertical pump adjacent each other.

For clearer understanding about the building's rigidity, Figure 11 viewing a graphical interpretation of structure rigidity on other spring-base value. It is founded that the structure would experience five times rotation generated from the same structure if it on a fixed-base footings.

This next analysis mainly as an expansion of EulerLagrangian approach, in Solidworks the approach algorithm is already built in within the Computational Fluid Dynamics (CFD) analysis feature. It is found that the fact of the fluid flow dynamics affected at impeller's force. To produce the effect of fluid dynamics at impeller force, several analysis conducted to describe a wider range of possibilities might occur at actual conditions.

From the Figure 12 and Figure 13, it can be concluded the actual rotation frequency acting on the vertical pump's is ranged between $88 \mathrm{rad} / \mathrm{s}$ to $120 \mathrm{rad} / \mathrm{s}$, or it is $14 \mathrm{~Hz}$ to $19 \mathrm{~Hz}$. These information significantly important for comparing the force intensity with buildings resonate frequency. Hence the collectible information of the geometric changing (Table 5) and the rotational frequencies (Figure 12 and Figure 13) it is possible to conduct the ODS simulation by using the information as an input load and initial geometric unbalanced, and harnessing the result from it. All ODS analysis then recorded at the place where the forces act (at the concrete overhunged beam). The Figure 14 viewing every fluctuate force (x-direction) in each pressure value (which corresponded to rotation frequency), and Figure 15 viewing the $y$-direction of fluctuate forces.

This final section shall discuss about NLTH analysis output, which came from the arbitrary loading data from Figure 12. Since the output is understand-able sinusoidal wave, hence the NLTH approach using feature from SAP2000 is considered reliable enough. 
With the comparison of fixed and spring conditions, the difference of both (beam) displacement value are displayed in graphical results below to see the behavior. At Figure 16, the displacement (x-direct.) from unbalance force (spring base), creates arbitrary wave with highdense fluctuates value alternating at $\mathbf{\pm 0 . 8} \mathbf{m m}$. And for the displacement from steady force (fixed base), it has value $\mathbf{\pm 0 . 0 6} \mathbf{m m}$ with near-zero excitation on it. This waves also seen at Figure 17 (y-direct. deflection) with another highdense fluctuates at value $\mathbf{+ 0 . 2} \mathbf{m m}$.

From the analysis output, hence some parametric study are conducted, and the following summary can be drawn:

1. Geometric changing in structural element of the building primary caused by the base rigidity. In this paper, the spring-base building experiencing nodal rotation 5 times larger than fixed-base building

2. Alternating in fluid pressure will significantly varying the rotation of the impeller, which affects the frequency state of the forces. This range of angular speed need to be mapped before designing the building, since from the Figure 18 shown that resonance occur at 14,5 Hertz.

3. Vibration phenomena occurrence at the building shall considered 'small' due to its comparisons with building's nominal strength, but in the perspective of mechanical parts this phenomena needs to have more attention since the concept of the building is 'nonstop' public civil service.

The author hopes the continuation of this study in order to eliminate most of the aforementioned limitations and model the problem in a more accurate manner. More cases of fairings and splitter plates will also be considered for the analysis at a later stage to better understand the FIV suppressing capabilities of different devices. From the above conclusions, the following recommendation can be drawn:

1. The pump station building need to be designed with higher range of natural frequencies. Since the geometric factor in structural response of the building takes significant account onto vibration phenomena.

2. Base rigidity holds siginificant factor to retain the geometric displacements, hence the installation of structural piles is mandatory since it will increase to a larger value of base rigidity.

3. The design phylosophy of the vertical pump are likely needs to be re-considered, eventhough it's well designed already. It is suggested to lowering the distance between pump and electric motor as Figure 19

4. The codes of structural design needs to consider the modal analysis into more specific tresholds. Especially if the building's structure is attached with machineries.

5. Further studies on the fluid-soil-structure interaction needed to dive deeper onto the magnification value of fluid and soil non-linearity.

\section{REFERENCES}

[1] E. Wrinkler, Die Lehre Von Elasticitaet Und Festigkeit, 1st ed. Prague: H-Dominicus, 1867.

[2] K. Terzhagi, "Evaluation of coefficients of subgrade reaction," Géotechnique, vol. 5, no. 4, pp. 297-326, 1955.

[3] A.B. Vesic, "Beams on Elastic Subgrade and Winkler's Hypothesis," in Proceedings of the 5th International Conference on Soil Mechanics and Foundation Engineering, 1961, pp. 845-850.

[4] J. E. Bowles, Foundation Analysis and Design, 5th ed. 1977.

[5] K. Chopra, Dynamics of Structures: Theory and Applications to Earthquake Engineering, 3rd ed. Singapore: McGraw-Hill Book, 2006.

[6] H. Koopman, "The vortex wakes of vibrating cylinders at low Reynolds numbers," J. Fluid Mech., vol. 28, pp. 501-512, 1967.

[7] C. Feng, "The measurement of vortex-induced effects in flow past stationary and oscillating circulator and D-section cylinder," University of British Columbia.

[8] A. Leonard, "Aspects of Flow-Induced Vibration," J. Fluid Struct., vol. 15, pp. 415-425, 2001.

[9] Y. U. Haiping and et al, "Sequential coupling simulation for electromagnetic-mechanical tube compression by finite element analysis," J. Mater. Process. Technol., vol. 209, pp. 707-713, 2009.

[10] Z. Benadla, "Influence of the soil-structure interaction on the seismic behavior of buildings on shallow foundations," Int. J. GEOMATE, vol. 6, pp. 811-816, 2014.

[11] J. Liang and et al, "Soil-structure interaction for a SDOF oscillator supported by a flexible foundation embedded in a half-space: Closed-form solution for incident plane $\mathrm{SH}$ waves," Soil Dyn. Earthq. Eng., vol. 90, pp. 287-298, 2016.

[12] G. Papazafeiropoulos and et al, "Dynamic Interaction of Concrete Dam-Reservoir-Foundation: Analytical and Numerical Solutions," Soil Dyn. Earthq. Eng., vol. 90, pp. 455-488, 2010.

[13] B. K. Kumar and et al, "Determination of Unbalance in Rotating Machine Using Vibration Signature Analysis," Int. J. Mod. Eng. Res., vol. 2, pp. 3415-3421, 2012.

[14] M.A. Saleem, "Detection of Unbalance in Rotating Machines Using Shaft Deflection Measurement during Its Operation," IOSR J. Mech. Civ. Eng., vol. 3, pp. 8-20, 2012.

[15] A. Zhang and et al, "Analysis of Nonlinear Vibration in Permanent Magnet Synchronous Motors under Unbalanced Magnetic Pull," J. Appl. Sci., vol. 8, no. 1, pp. 113-132, 2018. 